\title{
Analysis of Potential VLBI Southern Hemisphere Radio Calibrators
}

\author{
Aletha de Witt* and Michael Bietenholz \\ Hartebeesthoek Radio Astronomy Observatory \\ P.O. Box 443 \\ Krugersdorp 1740 \\ South Africa \\ E-mail: aletehartrao.ac.za
}

The Southern Hemisphere will see a major expansion in radio astronomy instruments in the future and this will dramatically increase the capabilities of the Southern Hemisphere VLBI array. The Southern Hemisphere VLBI array is presently not well served with calibrator sources and there are significant gaps in the calibrator distribution on the sky, especially at frequencies below $5 \mathrm{GHz}$. In order to address the problem of poor calibrator coverage of the Southern Hemisphere, dedicated surveys at $2.3 \mathrm{GHz}$ and $8.4 \mathrm{GHz}$ have been undertaken to observe radio sources using the Australian Long Baseline Array (LBA). The goal of these surveys is to find sources which are sufficiently compact to be suitable as VLBI calibrators. We present here a progress report on the imaging from two such surveys: the LBA calibrator survey (LCS), which is an ongoing project, as well as our own survey at $2.3 \mathrm{GHz}$. At $2.3 \mathrm{GHz}$, using VLBI observations of 57 candidate sources from the ATCA calibrator list, we identified 8 new sources suitable as VLBI calibrators at $2.3 \mathrm{GHz}$. At $8.4 \mathrm{GHz}$, we imaged a total of 122 calibrator sources from the on-going LCS in order to confirm their suitability as calibrators.

11th European VLBI Network Symposium \& Users Meeting,

October 9-12, 2012

Bordeaux, France

* Speaker. 


\section{Introduction}

The Southern Hemisphere is presently seeing a major increase in radio astronomy instruments and capabilities, with the building of the SKA-precursors, ASKAP (Australia) and MeerKAT (South Africa). In addition, there is also an effort underway to build an African VLBI network, mostly by converting old communication dishes in a number of African countries. The focus of the SKA precursors will be on frequencies below $5 \mathrm{GHz}$. The sky has been relatively little explored at high angular resolutions and frequencies $<1 \mathrm{GHz}$, and even less so in the Southern Hemisphere, therefore the coming of new VLBI stations opens up a significant "window of discovery". In order to fully utilize the capabilities of these instruments an adequately dense set of strong calibrator or reference sources that are compact on mas scales is needed. Currently, the density of VLBI calibrator sources shows a rapid drop in calibrator density at declinations south of $-30^{\circ}$. The LBA calibrator survey (LCS) was undertaken to address this deficit, and has already made a huge improvement at $8.4 \mathrm{GHz}$ [7]. However, detailed morphology information is not yet available for many of the LCS sources. At frequencies below $5 \mathrm{GHz}$, there is still a very significant deficit of VLBI calibrator sources at low declinations. Figure 1 shows the sky distribution of calibrator sources at 2.3 and $8.4 \mathrm{GHz}$ from the RFC VLBI Global Solution Catalogue ${ }^{1}$, which is the most complete catalogue of positions of compact radio sources.

In VLBI, calibrator sources are needed for phase referencing, which is required for imaging weak objects as well as for differential astrometry. A catalogue of compact radio calibrator or reference sources with positions known to an accuracy of several mas has a number of other applications including spacecraft navigation, and geodesy including monitoring the Earth's rotation.

What makes a good calibrator source? It should have an accurately known position and a constant and relatively high brightness at the frequency of observation so as to be detectable on all baselines, and, ideally, should be unresolved so as to look the same on all baselines. All real sources depart to some degree from this ideal, and it is important to determine the degree of this departure so as to assess the suitability of the source as a calibrator. Most VLBI calibrator sources are core dominated quasars with a small, faint jet. Sources which are dominated by an unresolved or almost unresolved core work well as calibrator sources. However, extragalactic radio sources are known to have extended emission and can display a variety of structure down to even mas scales. In addition, the structure may be different at different frequencies, so a good calibrator at one frequency may not be similarly good at another frequency. In addition to having non-point like structure, the vast majority of compact radio sources exhibit flux density variability on time-scales of years to weeks, and they also often display structural variability at mas scales over these same time-scales. These departures from the static point-source approximation introduce errors into the calibration of VLBI data, and can have a significant effect on the derived VLBI astrometry (e.g., $[1,2,4])$. It is important therefore to characterize the structure of potential calibrators not only at different frequencies but also at multiple epochs.

With the goal of increasing the Southern-Hemisphere VLBI calibrator source density, we (along with R. Booth, F. Hungwe, and C. Phillips) initiated a two-part survey of candidate sources from the ATCA calibrator list at $2.3 \mathrm{GHz}$, which had not previously been observed with VLBI be-

\footnotetext{
${ }^{1}$ The RFC VLBI Global Solution Catalogue is available on the web at http://astrogeo.org/vlbi/solutions/rfc_2013a; see also [7,8]
} 


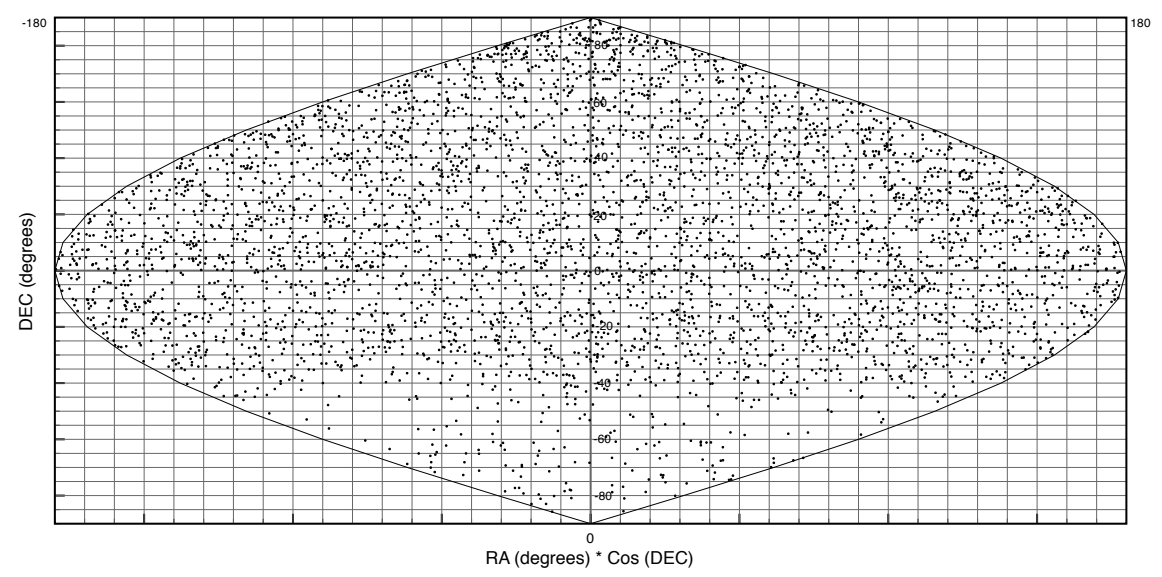

Figure 1: Sky distribution of 2.3- and 8.4-GHz calibrator sources from the VLBI Global Solution rfc_2011d Catalogue. The deficit of calibrators south of declination $-30^{\circ}$ can clearly be seen. Note that the LCS, which is still in progress, has already obtained a large number of additional calibrator sources in the region below declination $-30^{\circ}$.

low $5 \mathrm{GHz}$. We chose $2.3 \mathrm{GHz}$ because it is one of the two classic frequencies of the International Celestial Reference Frame (see [5]) and of many geodetic VLBI experiments.

Our strategy was first to make short VLBI observations of our selection of 56 candidate sources to determine which ones were too extended to be considered as VLBI calibrators, and then to obtain more extensive follow-up VLBI observations of the remainder to better characterize their structure. The follow-up observations had sufficient $u$ - $v$ coverage to produce high-quality $2.3 \mathrm{GHz}$ images, and reliably determine the source compactness and assess their suitability as calibrators.

In addition to the 2.3-GHz calibrator observations, we also imaged a selection of the observations from the LCS, which seeks to systematically observe southern flat-spectrum sources with VLBI and determine their positions with mas accuracy. To date the correlated flux densities and positions of 857 new objects among the 1083 scheduled for observations at $8.4 \mathrm{GHz}$ have been computed, and the astrometric results are made available on the web in the RFC VLBI Global Solution Catalogue mentioned above. In this progress report, we present the first imaging results from the LCS campaign.

\section{Observations at $2.3 \mathrm{GHz}$}

From the ATCA calibrator list, we selected 56 sources with flux densities $>350 \mathrm{mJy}$ at $2.3 \mathrm{GHz}$ and declinations $<-30^{\circ}$. Short observations of the 56 selected sources were made on 6 June 2008, using five of the LBA antennas; ATCA, Mopra, Parkes, Hobart and Ceduna, all in Australia. We observed at a frequency of $2.274 \mathrm{GHz}$, and recorded both senses of circular polarization (LCP and RCP; IEEE convention) with a total bandwidth of $32 \mathrm{MHz}$ per polarization. The observations consisted of one, 7-minute scan per target source. The observations were correlated at the Curtin University of Technology using the DiFX software correlator [3].

Results from the 2.3-GHz, 2008 observations showed a total of 24 sources to have flux densities $>200 \mathrm{mJy}$ and sizes $<10$ mas, making them ideal candidates for follow-up observations. 
These 24 sources were observed on 13 August 2011, using the same five antennas as above, as well as the $34 \mathrm{~m}$ and $70 \mathrm{~m}$ dishes at Tidbinbilla in Australia, and the dishes in Warkworth in New Zealand, and Hartebeesthoek in South Africa. We observed at a frequency of $2.285 \mathrm{GHz}$ with a total bandwidth of $32 \mathrm{MHz}$ per polarization. We recorded both RCP and LCP except at Tidbinbilla, where we recorded only RCP from the 70-m and only LCP from the 34-m. We obtained 3-8 scans of 5 minutes for each source. The observations were again correlated at the Curtin University of Technology using DiFX.

\section{Observations at $8.4 \mathrm{GHz}$ in 2010}

Observations for LCS experiment V271e, were made on 11-12 March 2010 using ATCA, Mopra, Parkes, Hobart and Ceduna. We observed at a frequency of $8.344 \mathrm{GHz}$, with a total bandwidth of $128 \mathrm{MHz}$, and recorded LCP. The experiment consisted of observations of 155 target sources, with 2-4 scans of 2-6 minutes duration per source. The data were correlated on the hardware geodetic correlator at Bonn.

\section{Data Reduction}

The data reduction for all three data sets was similar, and was done using AIPS. Amplitude gains were derived from system temperatures. Thereafter, data inspection, initial editing, and fringe fitting were done in the standard manner. We did an initial round of fringe-fitting to find approximate residual rates and delays. The main editing was carried out using this approximate calibration, and then using the edited data, we proceeded to a second round of fringe-fitting to refine the calibration, with each source being fringe-fitted individually. The visibility data were Fourier inverted and deconvolved using the CLEAN algorithm, and where possible, the amplitude gains were further refined by self-calibrating using CLEAN models [6].

Because of the limited, and sometimes highly elongated $u-v$ coverage of the initial $2.3-\mathrm{GHz}$ observations in 2008 , we did not attempt to characterize the source geometry beyond an estimate of the size, which was obtained by fitting a circular Gaussian model directly to the visibility data by least-squares. A circular Gaussian was chosen as it has the smallest number of parameters but will still give us an idea of the source angular size.

For the final CLEAN images from the 2011 2.3-GHz and 2010 8.4-GHz observations, we used complex weighting using the square root of the statistical visibility weights, which increases the robustness of the image. Although the $u-v$ coverage for the $8.4-\mathrm{GHz}$ data was generally sufficient for imaging, the sparse $u-v$ sampling is the most significant limitation on the image quality. Some sources were observed during only 2 scans and/or with fewer than 5 antennas, and to distinguish between real source structure and sidelobes often presented difficulties. In order to determine if any features aside from the central peak were real, we again fitted Gaussian models directly to the visibility data, starting with a single component model centred on the core object only, and then added further components to the model at the position of any other features in the image. If the multi-component model had a lower reduced Chi-squared than the single-component one, we take additional features in the images to be real. 


\section{Results}

We show representative contour plots of the images at 2.3 and $8.4 \mathrm{GHz}$ in Figure 2. Images and visibility plots can be obtained from the LCS website at http://astrogeo. org/lcs/cat/v271e_dew.html for all the sources at $8.4 \mathrm{GHz}$, and the $2.3-\mathrm{GHz}$ results will be uploaded soon. The total CLEAN flux densities of 2011 2.3-GHz sources range between 0.17 and $1.2 \mathrm{Jy}$, the image background rms between 1.03 and $8.94 \mathrm{mJy} \mathrm{beam}^{-1}$, and the fitted value of the beamsize between $\sim 2.5$ and $\sim 22$ mas. The total CLEAN flux densities of the 2010 8.4-GHz sources range between $\sim 0.025$ and $\sim 1.6 \mathrm{Jy}$ and the image background rms between 0.5 and $6.4 \mathrm{mJy}^{\mathrm{beam}}{ }^{-1}$, and the fitted beamsize of $\sim 4$ mas.

The images and the results from the model-fitting allowed us to get an idea of the source structure and we can loosely classify the sources into unresolved or almost unresolved sources, resolved sources and complex sources with a multi-component structure. We also compared the amplitude versus $u-v$ distance plots for each source with the results obtained from the imaging and modelfitting to determine the compactness of the sources and their usability as VLBI calibrators.

From the sources observed at $2.3 \mathrm{GHz}$ we found a total of eight new sources that can be used as calibrators for VLBI observations. Of these, one source appears compact on baselines of up to $80 \mathrm{M} \lambda$, six appear compact on baselines $\lesssim 18 \mathrm{M} \lambda$, and three appear compact on baselines $\lesssim 4 \mathrm{M} \lambda$.

The $u$-v coverage for the $8.4-\mathrm{GHz}$ observations from the LCS was not as good as would be obtained from dedicated imaging observations, and high-dynamic-range images are not possible for all the sources. The majority of the images, however, were good enough to determine source morphology. From the 122 sources observed at $8.4 \mathrm{GHz}$ in this particular LCS experiment, we imaged 117. Eight show structure which is extended on a scale of 10 mas or larger, and should be used only with caution as VLBI calibrators. The remainder appear to be dominated by a compact component, and should make good VLBI calibrators.

\section{Summary and Conclusions}

We present the first imaging results of two dedicated surveys with the aim of densifying the network of VLBI calibrators in the Southern Hemisphere. We identified eight new sources with declinations $<-30^{\circ}$ that can be used as VLBI calibrators at $2.3 \mathrm{GHz}$. We also confirmed the compactness of the majority of 8.4-GHz sources from the calibrator survey.

It should be noted however that the successful use of any source as a calibrator is conditional on the sensitivity, resolution, and frequency of the VLBI observations. We are currently in the process of re-calibrating and imaging the remainder of the 8.4-GHz sources from the LCS. We also aim to observe all the 8.4-GHz sources from the LCS at $2.3 \mathrm{GHz}$, to ensure that a dense net of calibrators is available also at $2.3 \mathrm{GHz}$. Furthermore, having observations at 2.3 and $8.4 \mathrm{GHz}$ will allow us to examine the frequency-dependence of the source structure for a large sample of extragalactic sources.

\section{Acknowledgements}

The Authors wish to thank RadioNet3 for the financial support to attend the conference. 


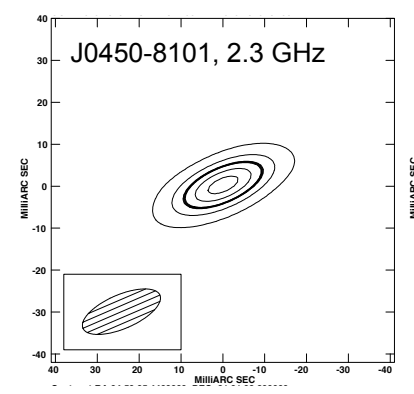

Peak flux $=0.908 \mathrm{Jy} /$ beam

$\mathrm{rms}=2.70 \mathrm{mJy}$

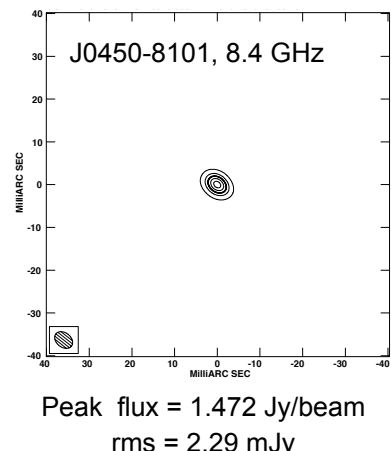

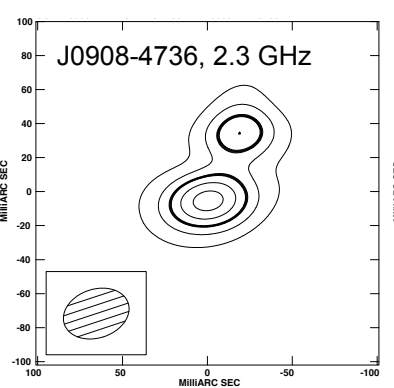

Peak flux $=0.317 \mathrm{Jy} /$ beam $\mathrm{rms}=8.19 \mathrm{mJy}$

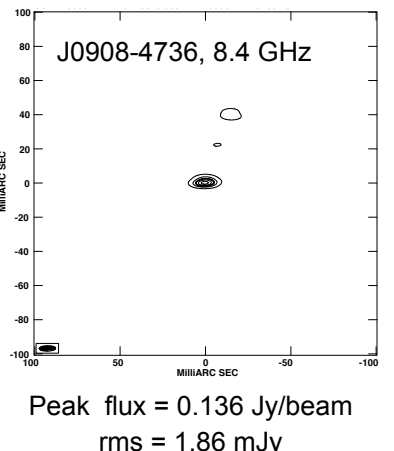

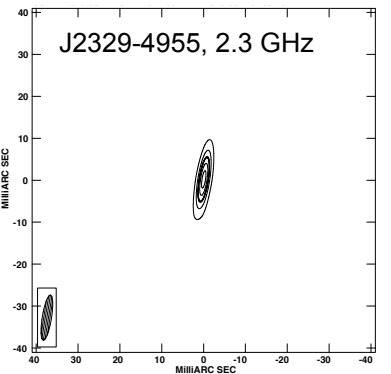

Peak flux $=0.119 \mathrm{Jy} /$ beam $\mathrm{rms}=3.13 \mathrm{mJy}$

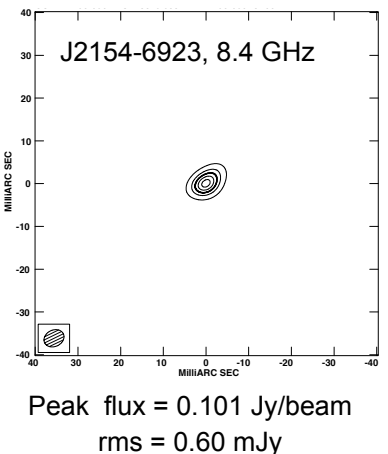

Figure 2: Maps of sources at 2.3 and $8.4 \mathrm{GHz}$ from the LBA calibrator surveys. Contour levels are at -10 , $10,30,50,70$ and $90 \%$ of the peak brightness. The $50 \%$ contour is emphasized. North is up and East is to the left. The source name, frequency, peak brightness and background rms levels are noted in each panel.

\section{References}

[1] N. Bartel, M. F. Bietenholz, D. E. Lebach, J. I. Lederman, R. R. Ransom, M. I. Ratner, L. Petrov, VLBI for Gravity Probe B. III. A Limit on the Proper Motion of the "Core" of the Quasar 3C 454.3, ApJS, 201 (2012), 3

[2] P. Charlot, Radio-Source Structure in Astrometric and Geodetic Very Long Baseline Interferometry, AJ, 99 (1990), 1309

[3] A. T. Deller, S. J. Tingay, M. Bailes, C. West, DiFX: A Software Correlator for Very Long Baseline Interferometry Using Multiprocessor Computing Environments, PASP, 119 (2007), 318

[4] A. L. Fey, P. Charlot, VLBA Observations of Radio Reference Frame Sources. II. Astrometric Suitability Based on Observed Structure, ApJS, 111 (1997), 95

[5] A. L. Fey, D. Gordon, C. S. Jacobs (eds.), The Second Realization of the International Celestial Reference Frame by Very Long Baseline Interferometry, IERS Technical Note 35, 2009

[6] T. J. Pearson, A. C. S. Readhead, Image Formation by Self-Calibration in Radio Astronomy ARA\&A, 22 (1984), 97

[7] L. Petrov, C. Phillips, A. Bertarini, T. Murphy, E. M. Sadler, The LBA Calibrator Survey of southern compact extragalactic radio sources - LCS1, MNRAS, 414 (2011), 2528

[8] L. Petrov, The EVN Galactic Plane Survey - EGaPS, MNRAS, 419 (2012), 1097 